\title{
Wavelength Selection Criteria and Link Availability due to Cloud Coverage Statistics and Attenuation affecting Satellite, Aerial, and Downlink Scenarios
}

\author{
Florian Moll ${ }^{* a}$, Markus Knapek ${ }^{\mathrm{a}}$ \\ ${ }^{a}$ German Aerospace Center (DLR), Institute for Communications and Navigation, Oberpfaffenhofen, \\ Germany
}

\begin{abstract}
The choice of wavelength is essential for the variety of different communication scenarios in the field of free space optics (FSO). Possibilities are Satellite and HAP (High Altitude Platform) Downlinks, HAP-HAP links, HAP-Satellite links and all kinds of links involving aeronautical vehicles. This paper addresses the influence of the wavelength dependent attenuation of clouds, the atmospheric transmission in the NIR and MIR and a statistical analysis of cloud coverage data for an estimation of link availability. Regarding the calculation of atmospheric transmission the free available simulation tools libRadtran and GENLN2 have been used. To identify advantageous wavelengths to increase link availability, cloud attenuation is determined by Mie scattering calculations of particle size distributions of various cloud types. Here the MIR wavelength interval between $10 \mu \mathrm{m}$ and $12 \mu \mathrm{m}$ has been found to give the lowest attenuation in clouds. However in most cases clouds will block the optical link. For that matter a statistical analysis of satellite based data from the European Cloud Climatology (ECC) is done to reveal favorable places with high availability in Europe. The improvement of link availability when a concept of ground station diversity is applied has also been investigated. An availability of almost $99 \%$ is reached with four hypothetical stations in southern Europe. Further the difference between availability values of single years decreases with multiple stations.
\end{abstract}

Keywords: Atmospheric attenuation, cloud attenuation, ECC, free space optics, GENLN2, link availability, MIR, statistical cloud coverage, libRadtran

\section{INTRODUCTION}

The atmospheric attenuation of a collimated light beam means a reduction of intensity in the direction of propagation. In physical terms this is also called the extinction of the beam. This comprises the absorption and the scattering. If the attenuation is independent of intensity the atmospheric attenuation can be described with Beer's law. It determines the attenuation of a path through the atmosphere by using the medium dependent extinction coefficient $\alpha_{\mathrm{ext}}\left[\mathrm{km}^{-1}\right]$ and the length $L[\mathrm{~km}]$. Therefore it is very important for the availability of the optical link especially when the medium is a cloud. It shows an exponential relation of the output intensity $I\left[\mathrm{~W} / \mathrm{m}^{2}\right]$ after the path $L$ and the input intensity $I_{0}\left[\mathrm{~W} / \mathrm{m}^{2}\right]$ over $\alpha_{\text {ext }}$ which only dependents on the wavelength $\lambda$ when the medium is assumed homogenous and the light monochromatic.

$$
I=I_{0} \cdot \exp \left(-\alpha_{\text {ext }}(\lambda) \cdot L\right)
$$

For the different free space optical communication (FSOC) scenarios the propagation path has different characteristics. So the choice of wavelength has to be made for all scenarios separately. Because plain space links like from a LEO (Low Earth Orbit) to GEO (Geosynchronous Earth Orbit) don't suffer from atmospheric effects a short wavelength should be used to minimize free space loss. Same counts for stratospheric HAP-HAP-links (HAP - High Altitude Platform) because these hover over the cloud layer. The most affected path is the one that includes propagation through all atmospheric layers and thus through all possible cloud layers, e.g. an OGS-LEO-link (OGS - Optical Ground Station) or an OGS-HAP-link. Because cloud attenuation in general is quite high and blocks the link completely, a statistical approach must be made to identify proper OGS locations for that case. When no location with sufficiently high availability can be found, multiple ground stations can be used to form a ground station diversity system.

\footnotetext{
*florian.moll@dlr.de
}

Copyright 2007 Society of Photo-Optical Instrumentation Engineers. This paper was published in SPIE Free-Space Laser Communications VII and is made available as an electronic reprint with permission of SPIE. One print or electronic copy may be made for personal use only. Systematic or multiple reproduction, distribution to multiple locations via electronic or other means, duplication of any material in this paper for a fee or for commercial purposes, or modification of the content of the paper are prohibited. 
The wavelength selection criterion addressed is cloud attenuation as low as possible. To raise availability for the case of links influenced by clouds long wavelengths should be preferred because they scatter less under presence of small particles. The limit for usable long wavelengths is given by the atmospheric window VIII between $7.5 \mu \mathrm{m}$ and $15.0 \mu \mathrm{m}$ as it is defined in [1]. All the atmospheric windows are shown in Fig. 1. The graph is the result of a low resolution calculation with the software suite libRadtran by using the LOWTRAN (LOW TRANsition code) model [2]. Over $15.0 \mu \mathrm{m}$ the atmospheric absorption does not allow transmission anymore. The goal of this paper is to identify wavelengths with low cloud attenuation and to show the availability of single ground stations and a system with ground station diversity in Europe. For that matter calculations of Mie scattering of various water cloud models are done because these cloud types are the ones with high attenuation. Ice clouds have much less attenuation and are almost wavelength independent because of their high particle radius. The problem with these cloud types is a depolarisation effect on the scattered wave, but this is not a problem which is addressed here. For details about propagation through ice clouds see [4]. The availability of FSOC systems is estimated by using satellite image data of Europe.

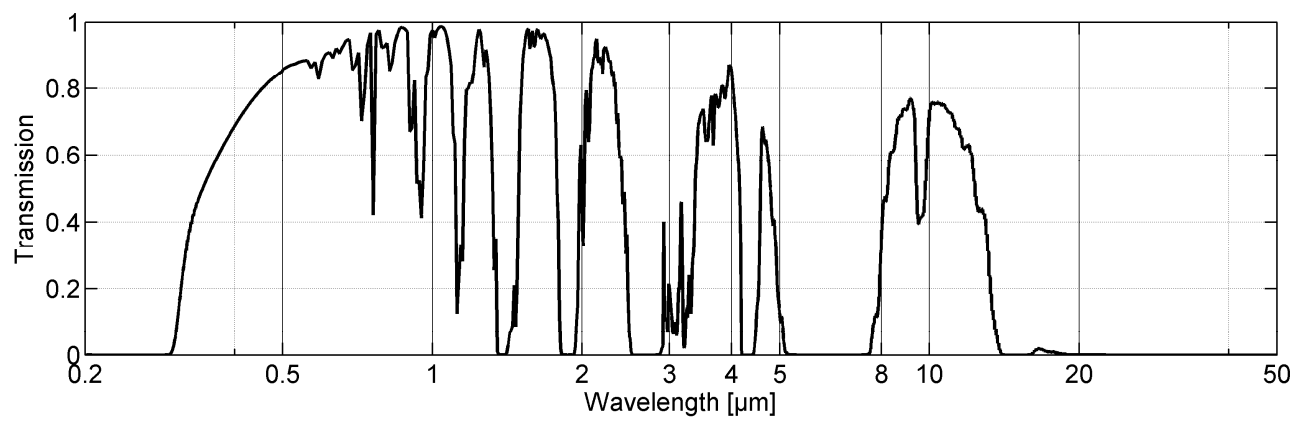

Fig. 1. Atmospheric transmission in the VIS, NIR and MIR calculated with libRadtran using the LOWTRAN model. The atmospheric windows are clearly visible. For low cloud attenuation window VIII $(7.5 \mu \mathrm{m}-15.0 \mu \mathrm{m})$ is of major concern.

\section{CLOUD ATTENUATION}

\subsection{Modelling of clouds}

For the calculation of the wavelength dependent attenuation of a cloud it is necessary to describe this cloud with a proper model. This comprises the particle size distribution of the water drops in the cloud and the respective wavelength dependent complex refraction index $n$. The shape of the particles is assumed to be spherical. Further the geometrical extent of the cloud in vertical direction is needed. The horizontal extent is not important because attenuation woll be given for vertical propagation. For simplicity the cloud is regarded homogenous and not polluted. With these constraints it is possible to set up a simple to use cloud model.

A commonly used function that describes the particle size distribution is the one proposed by Dermendjian in 1969 and is called the modified gamma function $f(r)$. It has the form

$$
f(r)=a \cdot r^{\alpha} \cdot \exp \left(-b \cdot r^{\gamma}\right) .
$$

where $r[\mu \mathrm{m}]$ is the drop radius and the four variables $a, \alpha, b$ and $\gamma$ are cloud type dependent empirical parameters [3]. A list of these parameters for several kinds of clouds is given in [5]. A somewhat different notation of equation (2) is used by K. N. Liou in [6] but the principal course of the function doesn't change. Here only the absolute number of particles per unit volume and the mode radius $r_{m}$ - the radius of the most frequent drop size - is needed for parameterization. Thus cloud models can be created with less effort but lower fit to measured size distributions. The drop size distributions of a Stratus and Stratocumulus cloud in Fig. 2 are generated with equation (2) using the same parameterization as in LOWTRAN and FASCODE [5]. The first one has a mode radius of $3.33 \mu \mathrm{m}$ and the second one $2.67 \mu \mathrm{m}$.

Stratus and Stratocumulus clouds belong to the family of low clouds. All clouds are divided into four groups with several different cloud types. In middle latitudes as in Europe, low clouds are defined by the international cloud atlas from 0$2 \mathrm{~km}$ height, middle clouds from 2-7 km and high clouds from 5-13 km [7]. A forth group - but not defined in [7] - are 
vertical clouds which can have an extent from near ground to the cloud maximum height of $13 \mathrm{~km}$. The groups are associated to the cloud types as follows:

- Low

Stratus, Stratocumulus

- Middle

Altocumulus, Altostratus

- High

Cirrus, Cirrostratus, Cirrocumulus

- Vertical

Nimbostratus, Cumulonimbus

The geometrical extent of these cloud types differs and ranges from a few hundred meters to several kilometers. Stratus and Stratocumulus range from $0.2 \mathrm{~km}$ to $0.8 \mathrm{~km}$, Altostratus from $0.2 \mathrm{~km}$ to $2 \mathrm{~km}$, Nimbostratus from 2 to $3 \mathrm{~km}$, Cirrus from 1.0 to $2.5 \mathrm{~km}$ and Cumulus from 0.5 to $5 \mathrm{~km}[8]$.

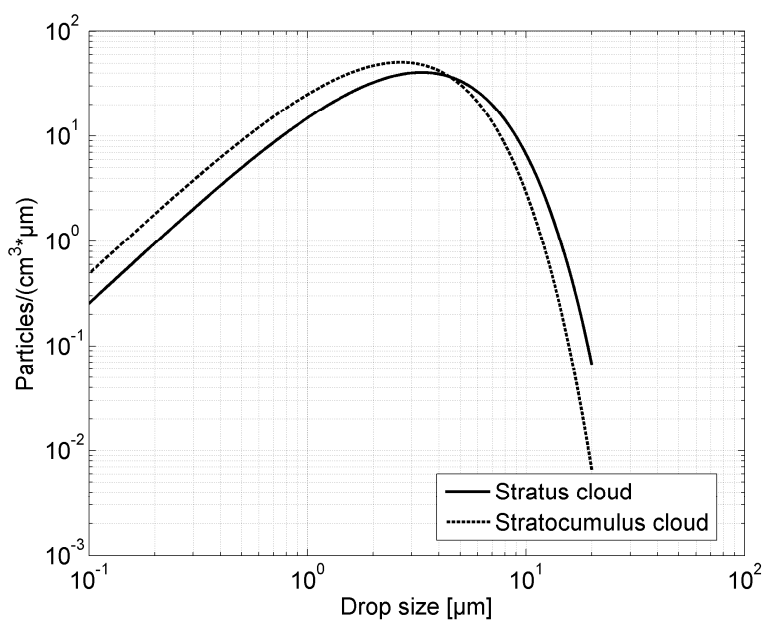

Fig. 2. Drop size distribution for the Stratus and Stratocumulus cloud type. The first one has a mode radius of $3.33 \mu \mathrm{m}$ and the second one $2.67 \mu \mathrm{m}$. These distributions are generated with the modified gamma function of Dermendjian using the same parameterization as in LOWTRAN and FASCODE.

\subsection{Mie Calculation with libRadtran}

To determine the attenuation of a cloud - regarded as a medium with specific particle size distribution - a Mie calculation for every drop size in the wavelength spectrum of interest has to be performed. The theory of Mie regards every particle separately and calculates the absorption and scattering habit. These two comprise the extinction of a single particle. For characterization of the extinction of a single particle the extinction efficiency $Q_{\mathrm{e}}$ is introduced. This is the scattering cross section of the particle $\sigma_{\mathrm{e}}\left[\mathrm{m}^{2}\right]$ over its geometrical cross section $\sigma_{\mathrm{g}}\left[\mathrm{m}^{2}\right]$ and thus is dimensionless.

$$
Q_{\mathrm{e}}=\frac{\sigma_{\mathrm{e}}}{\sigma_{\mathrm{g}}} .
$$

The scattering cross section is calculated with Mie's formula of the complex scattered field. Just as for the extinction a scattering efficiency $Q_{\mathrm{s}}$ and absorption efficiency $Q_{\mathrm{a}}$ are defined as expressed in equation (4). For a detailed description see [9].

$$
Q_{\mathrm{e}}=Q_{\mathrm{s}}+Q_{\mathrm{a}}
$$

When the wavelength is much bigger than the particle dimension the extinction is described by Rayleigh scattering which is a special case of the Mie scattering. Thus it is expected that with increasing wavelength the extinction decreases. However this is not the case for all wavelengths which is shown in Fig. 3. By looking at the course of $r=4 \mu \mathrm{m}$ and $10 \mu \mathrm{m}$ strong oscillations are observable in the lower spectrum which cause the extinction to change rapidly. In the high spectrum where the extinction should decrease several maxima can be seen at the wavelengths where 
the imaginary part of the complex refraction index is high and thus the absorption of the particle also is. This is especially bold around $20 \mu \mathrm{m}$. A distinctive local minimum exists between $10 \mu \mathrm{m}$ and $12 \mu \mathrm{m}$. This is because a low scattering efficiency meets low absorption efficiency in this interval. Beyond $50 \mu \mathrm{m}$ the extinction efficiency monotonic decreases and thus does the attenuation of the clouds. But in the longer wavelength starting from $20 \mu \mathrm{m}$ the molecular absorption of water increases expensively and no transmission windows exist. Thus the wavelengths over $20 \mu \mathrm{m}$ are not feasible.

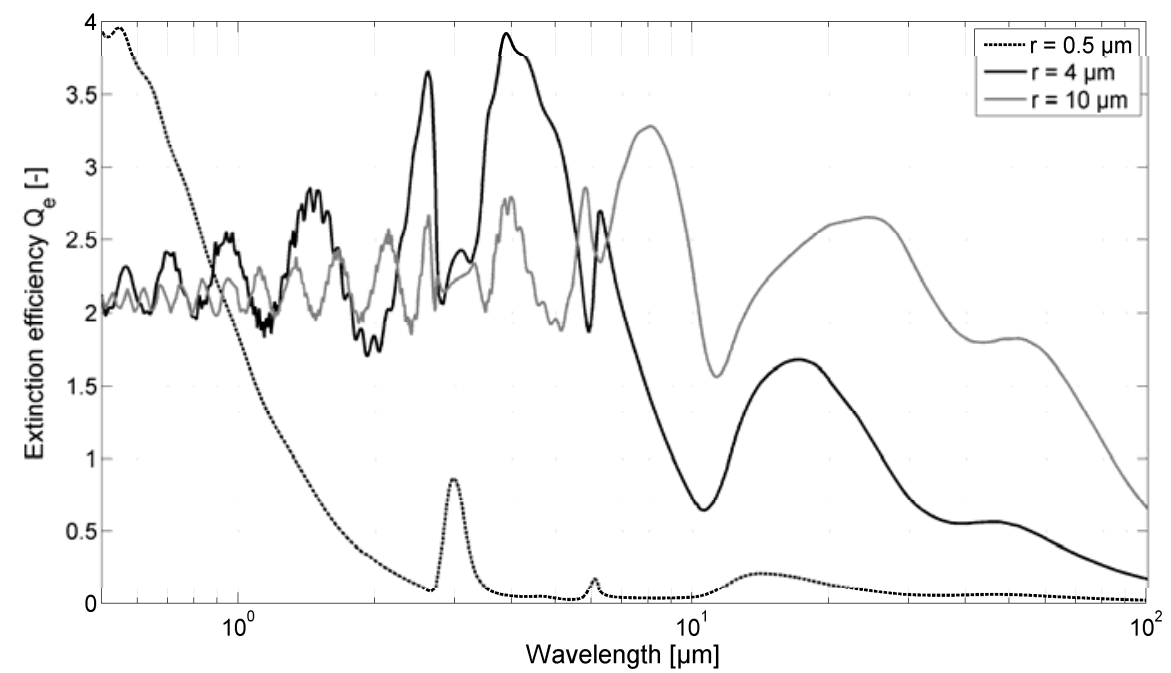

Fig. 3. Mie extinction efficiency over wavelength for three particle sizes. When the particle radius is in the same order of magnitude as the wavelength strong oscillations are observable. A distinctive local minimum of the extinction lies between $10 \mu \mathrm{m}$ and $12 \mu \mathrm{m}$ which is mainly caused by the characteristic of the wavelength dependent complex refraction index.

Using the Mie efficiency the extinction coefficient is calculated with [9]

$$
\alpha_{\text {ext,cloud }}(\lambda)=10^{3} \cdot \int_{0}^{\infty} f(r) Q_{\mathrm{e}}(\lambda, r, n) \pi r^{2} d r \quad\left[k m^{-1}\right] .
$$

The extinction coefficient of the cloud $\alpha_{\text {ext,cloud }}$ is then put in the law of Beer which results in the attenuation of the cloud.

Because a cloud exists of many particles of different size which all have their contribution to the total attenuation the calculation is somewhat time-consuming. The calculations are done with the Mie tool included in the software suite libRadtran. This is developed for the calculation of radiation transfer in the atmosphere. The Mie tool takes a specific particle size distribution and complex refraction index as input and calculates the total extinction coefficient per unit concentration. By applying Beer's law the cloud attenuation in $[\mathrm{dB} / \mathrm{km}]$ can be calculated. The result is shown in Fig. 4 . Five cloud types are compared. All clouds show roughly the same course. In the VIS and NIR the attenuation increases almost linearly except for a few local minima and maxima. The most interesting are the local minima between $10 \mu \mathrm{m}$ and $12 \mu \mathrm{m}$. Because no appropriate transmission window over $15 \mu \mathrm{m}$ exits, the wavelength with lowest cloud attenuation has to be searched between $10 \mu \mathrm{m}$ and $12 \mu \mathrm{m}$. The cloud with the highest attenuation is the Cumulus type. Is also has the highest mode radius and with an absolute particle number of $N=250 \mathrm{~cm}^{-3}$ also the highest liquid water content $W$. Because Cumulus clouds are within an extent of 0.5 to $5.0 \mathrm{~km}$ the attenuation will be too high for all wavelengths. Same counts for Nimbostratus $(2 \mathrm{~km}$ to $3 \mathrm{~km})$ and Altostratus $(0.2 \mathrm{~km}$ to $2.0 \mathrm{~km})$. The attenuation roughly decreases with lower liquid water content.

Stratus and Stratocumulus have the lowest attenuation per $\mathrm{km}$ and extent $(0.2 \mathrm{~km}$ to $0.8 \mathrm{~km})$ and thus are worth to be examined in more detail. For the minimal and maximal extents the total attenuation is calculated and shown in Fig. 5. This gives an idea of the attenuation dynamic of the Stratus and Stratocumulus. In the VIS and NIR the dynamic is about $150 \mathrm{~dB}$ for the first and $90 \mathrm{~dB}$ for the latter. In both cases for a minimal cloud thickness of $200 \mathrm{~m}$ the attenuation exceeds $30 \mathrm{~dB}$ in this spectral region. In the interval of the local minimum between $10 \mu \mathrm{m}$ and $12 \mu \mathrm{m}$ the attenuation has an 
acceptable order of magnitude. Considered are now the lowest possible cloud attention when $\mathrm{z}=200 \mathrm{~m}$. A Stratus cloud reaches a minimal attenuation of $29 \mathrm{~dB}$ at $\lambda_{\text {min }}=11.13 \mu \mathrm{m}$, the Stratocumulus cloud $15.8 \mathrm{~dB}$ at $\lambda_{\min }=11.05 \mu \mathrm{m}$. These are minimum values which are exceeded most of the time. The maximum values for $\mathrm{z}=800 \mathrm{~m}$ are $116.1 \mathrm{~dB}$ and $63.3 \mathrm{~dB}$ respectively. The remaining cloud types in Fig. 4 are not discussed in more detail because their total attenuation is found out to be much too high for any wavelength due to their large vertical extent and high attenuation per km.

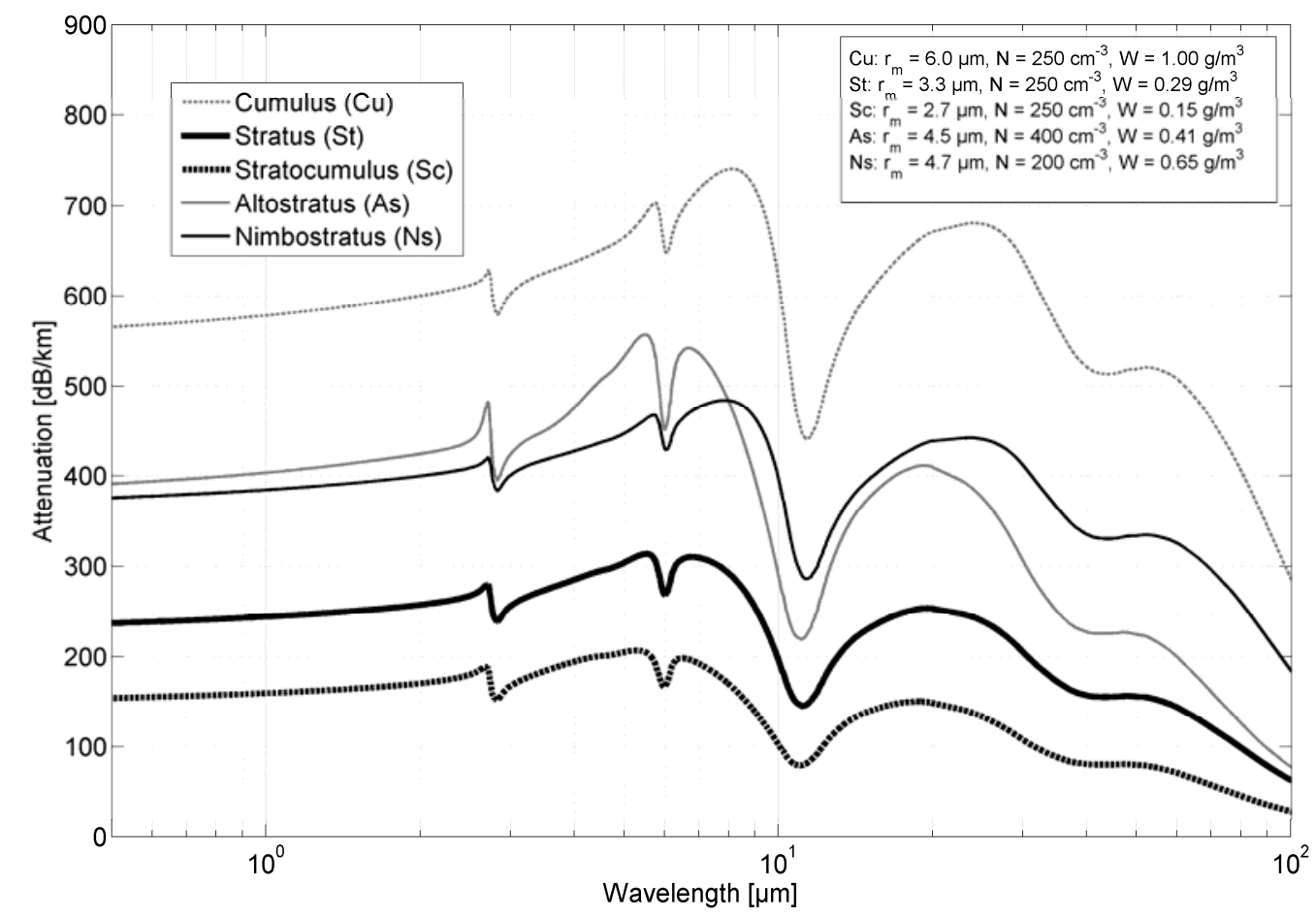

Fig. 4. Attenuation per km of five cloud types. All types show roughly the same course. Eye catching is the local minimum between $10 \mu \mathrm{m}$ and $12 \mu \mathrm{m}$. So wavelengths must be looked for in this spectral interval. The text box contains the microphysical parameters of the cloud types as the mode radius $r_{\mathrm{m}}$, the particle concentration $N$ and the liquid water content $W$.
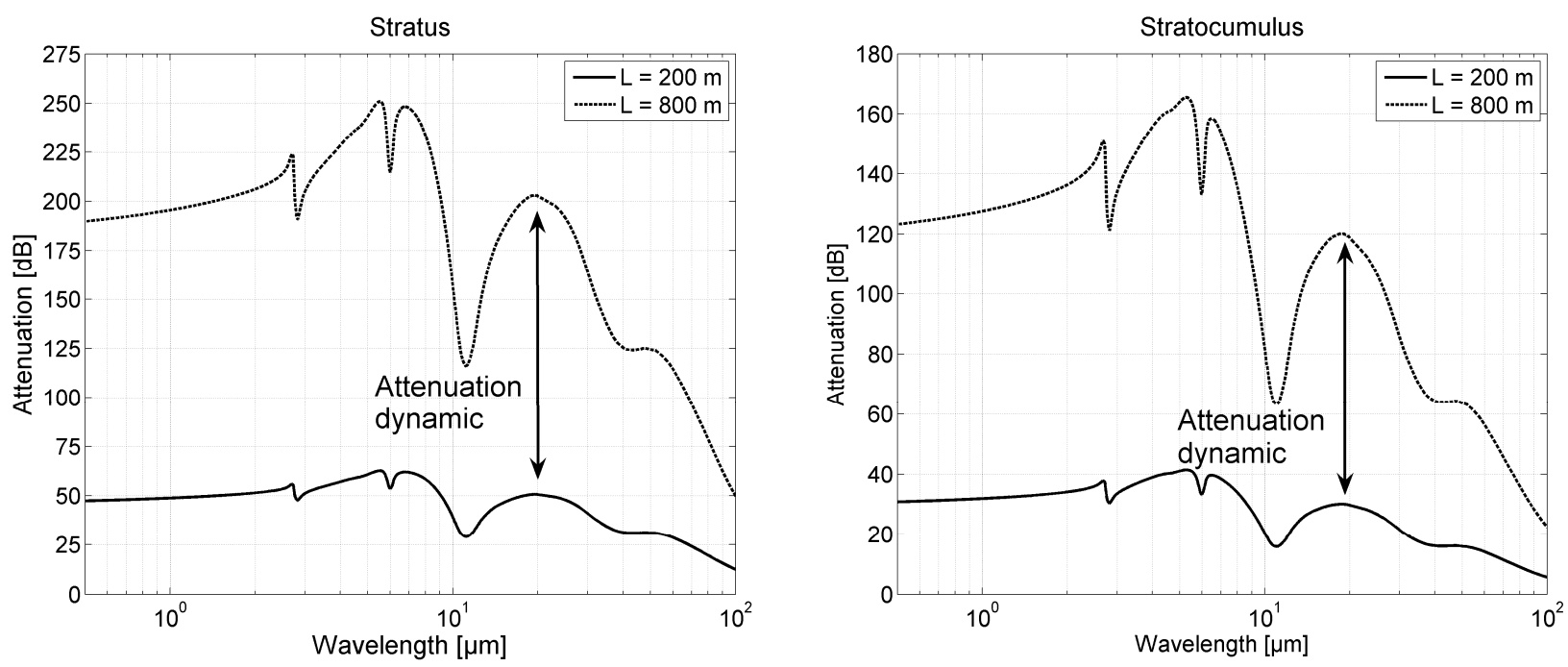

Fig. 5. Dynamics of absolute attenuation of Stratus and Stratocumulus clouds. The solid lines stand for the attenuation with vertical thickness of $800 \mathrm{~m}$, the dashed lines for $200 \mathrm{~m}$. The local minimum of the Stratus is at $\lambda_{\min }=11.13 \mu \mathrm{m}(29.0 \mathrm{~dB})$, the one of the Stratocumulus at $\lambda_{\text {min }}=11.05 \mu \mathrm{m}(15.8 \mathrm{~dB})$. 


\section{WAVELENGTH SELECTION FOR BETTER CLOUD TRANSMISSION}

The results of the Mie calculations reveal the interesting spectral interval between $10 \mu \mathrm{m}$ and $12 \mu \mathrm{m}$. In Table $1 \mathrm{a}$ comparison of the attenuation $A_{\mathrm{dB}}[\mathrm{dB}]$ of chosen cloud and fog models is presented. Considering the Stratus and Stratocumulus, the wavelengths $1060 \mathrm{~nm}$ and $1550 \mathrm{~nm}$ suffer from nearly the same attenuation of about $50 \mathrm{~dB}$. A reduction of attenuation of almost $20 \mathrm{~dB}$ is achieved by applying a $\mathrm{CO}_{2}$-Laser. It has the potential to provide sufficient power and stability for use in FSOC. A somewhat different behavior is observed with the fog types. Whereas moderate radiation fog does not cause severe problems, heavy advection fog attenuates strongly because of its high water content. The attenuation is even higher by use of a $\mathrm{CO}_{2}$-Laser because the particle size is in the magnitude of its wavelength $\left(r_{\mathrm{m}}=10 \mu \mathrm{m}\right)$ and thus Mie scattering is quite high. So to take a benefit out of the detected local attenuation minima, it is not possible to declare a single wavelength to be most suitable. Either a tunable laser source that can be adjusted in the range between $10 \mu \mathrm{m}$ and $12 \mu \mathrm{m}$ must be used or a compromise has to be made.

Table 1. Attenuation $A_{\mathrm{dB}}$ of different cloud types for various wavelengths that are considered in FSO contrasting the wavelength of the respective local minimum.

\begin{tabular}{|l|l|l|l|l|}
\hline Cloud type & $\begin{array}{l}A_{\mathrm{dB}}(1064 \mathrm{~nm}) \\
(\mathrm{Nd}: \mathrm{YAG})\end{array}$ & $\begin{array}{l}A_{\mathrm{dB}}(1550 \mathrm{~nm}) \\
(\mathrm{InGaAs})\end{array}$ & $\begin{array}{l}A_{\mathrm{dB}}(10.6 \mu \mathrm{m}) \\
\left(\mathrm{CO}_{2}\right)\end{array}$ & $\begin{array}{l}A_{\mathrm{dB}} \\
\left(\lambda_{\min }\right)\end{array}$ \\
\hline Stratus $(200 \mathrm{~m})$ & 49.0 & 50.3 & 31.2 & $29.0(11.13 \mu \mathrm{m})$ \\
\hline Stratocumulus $(200 \mathrm{~m})$ & 32.0 & 33.0 & 16.5 & $15.8(11.05 \mu \mathrm{m})$ \\
\hline Heavy advection fog $(100 \mathrm{~m})$ & 12.6 & 12.8 & 13.6 & $11.6(11.60 \mu \mathrm{m})$ \\
\hline Moderate radiation fog $(100 \mathrm{~m})$ & 3.9 & 4.3 & 0.8 & $0.8(10.40 \mu \mathrm{m})$ \\
\hline
\end{tabular}

So far wavelengths with low minimal cloud attenuation are identified, but the atmospheric attenuation still has to be examined. Because of strong thin attenuation lines in the MIR that are caused by molecular absorption of $\mathrm{H}_{2} \mathrm{O}$ and $\mathrm{CO}_{2}$ a high resolution calculation of the atmospheric attenuation must be done to resolve these lines. This calculation can be performed with the software tool GENLN2 [10] which uses HITRAN (HIgh-resolution TRANsmission molecular absorption data base) as a direct input. HITRAN consists of several spectroscopic parameters which describe the absorption lines of the air molecules and which are needed to perform a high resolution calculation of the atmospheric absorption. The spectral interval between $7 \mu \mathrm{m}$ and $15 \mu \mathrm{m}$ is shown in Fig. 6. Scattering of aerosols and air molecules (Rayleigh scattering) is neglected in this plot because it is quite low in the considered spectral region and thus only the strong absorption lines determine the choice of wavelength. The left plot covers the whole atmospheric window VIII and illustrates quite clearly the importance of the high resolution calculation because of the many interruptions of the transmission window which are lost in the plot in Fig. 1. The right graph shows a zoom of the interval between $10.5 \mu \mathrm{m}$ and $11 \mu \mathrm{m}$ where the position of the $\mathrm{CO}_{2}$-Laser is tagged. Problems with tuning arise by meeting the absorption lines.
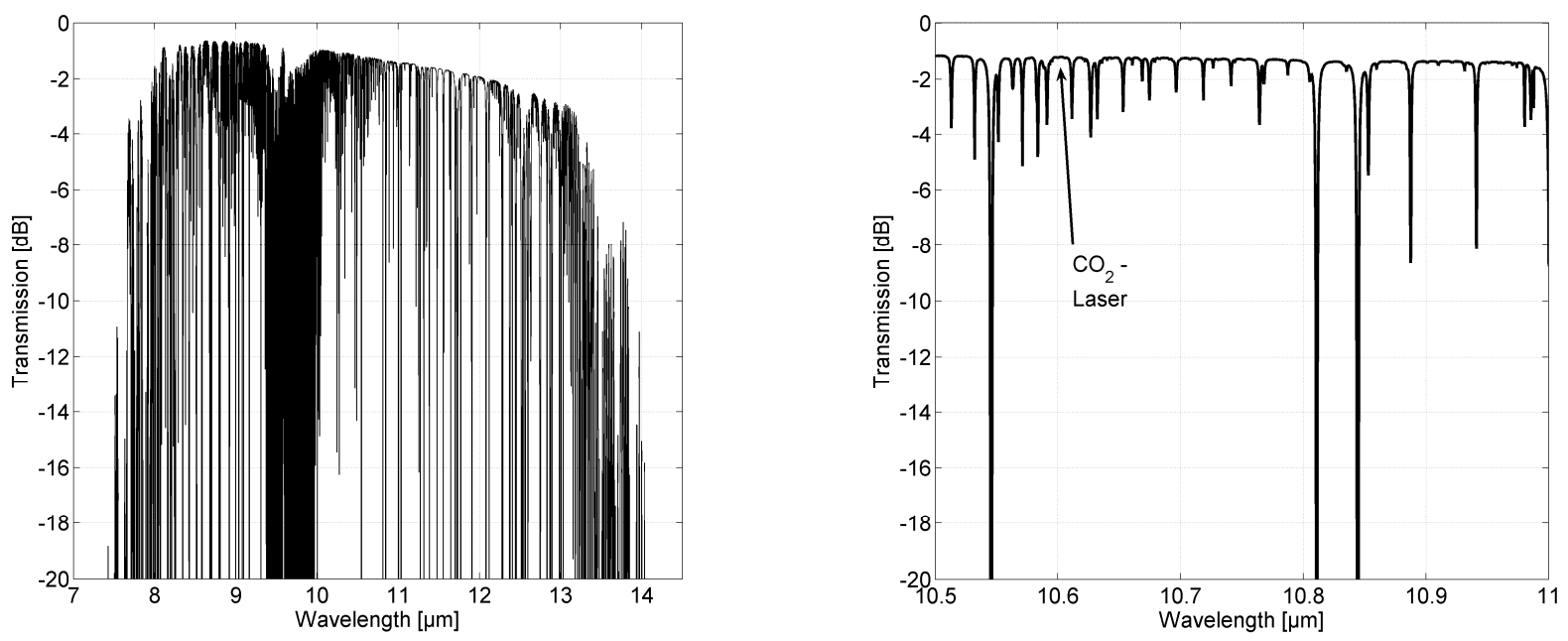

Fig. 6. Atmospheric transmission in vertical direction simulated with GENLN2 exclusively regarding molecular absorption. The used atmospheric model is Midlatitude summer. The left graph shows the whole atmospheric window VIII as defined in [1], the right one the zoomed region between $10.5 \mu \mathrm{m}$ and $11 \mu \mathrm{m}$. The emission wavelength of the $\mathrm{CO}_{2}$-Laser lies between the absorption lines of $\mathrm{H}_{2} \mathrm{O}$. Because in the depicted wavelength interval Rayleigh scattering is negligible and Mie scattering of aerosols (not clouds and fog) is low too, this graph also gives approximately the real attenuation. 


\section{LINK AVAILABILITY ESTIMATED FROM CLOUD COVERAGE STATISTICS}

\subsection{Avoiding the cloud problem}

The Chapters 2 and 3 have shown that it is very difficult to decrease cloud attenuation with wavelength selection. Thus in the following it is assumed that all cloud occurrence blocks the optical link. From satellite images the availability of the communication system is determined with statistics of the cloud coverage.

\subsection{Data base}

The statistics described here are derived from data from the European Cloud Climatology (ECC) Project. The images are taken by the AVHRR/3 (Advanced Very High Resolution Radiometer Version 3) which is the payload of the NOAA satellites. At the time of evaluation five years of ready processed data were available. These are the years 1990, 1995, 2000, 2004 and 2005. The ECC map covers almost whole Europe. It goes from $34^{\circ} \mathrm{N}$ to $72^{\circ} \mathrm{N}$ and from $11^{\circ} \mathrm{W}$ to $32^{\circ} \mathrm{E}$. The flyover time of the satellites ranges from 8 am to $5 \mathrm{pm}$. Thus values from different times must be compared. Meerkötter et al. found out that diurnal changes of the monthly mean cloud cover are relatively small. So by comparing different times on different days the time dependent cloud cover is averaged out, but statements about spatial coverage can be done. The resolution of the ECC data is $1.1 \mathrm{~km} \times 1.1 \mathrm{~km}$ in nadir direction. At the edges of the satellite images the resolution can degrade down to $6 \mathrm{~km}$ [11] [12].

\subsection{Availability of single ground stations}

For several time periods the mean cloud coverage is determined. The more values are available for the calculation of a mean value the more reliable it becomes. So as much images as possible are used. When an image is taken at a particular overfly it doesn't cover the whole ECC map. So when building an average of a certain amount of images one must care of the weighting for not falsifying the result. Also not every day the amount of images is the same. To minimize this influence a weighted mean is calculated where at first means are calculated for the single months and then comprise the final mean $P(W)_{\text {Year. }}$.

$$
P(W)_{\text {Year }}=\frac{1}{K} \sum_{i=1}^{K} P(W)_{\text {Month }, i} .
$$

Here the number of months is $K$ and $P(W)_{\text {Month }}$ is the mean value of a single month (the frequency of cloud occurrence in that month). The mean availability over the year $P(V)_{\text {Year }}$ is then 1-P(W) Year. The amount of images for a month mean varies between 50 and 150 depending on the satellites which delivered pictures in this month. The mean cloud coverage is shown in Fig. 7, left. The typical north-south decrease is clearly visible. In Germany the mean availability varies between $30 \%$ and $50 \%$. In latitudes over $60^{\circ} \mathrm{N}$ only availabilities smaller $40 \%$ are expected. Below $45^{\circ} \mathrm{N}$ better values are observed and availabilities over $70 \%$ can be reached. At the Alps and Carpathians local weather effects and obviously more frequent cloud occurrence can be observed. Thus it cannot be said generally that an OGS on mountains suffers less from cloud occurrence because some types of clouds lie beneath it. The availability in southern Europe is better than in the northern part, which is confirmed in Fig. 7, right. Here the ECC map is divided into an area with availability over and below $60 \%$. In Table 2 values for chosen OGS locations are given. These are locations where already satellite ground stations or astronomical telescopes are situated except for the location Aix-en-Provence near Marseille which is chosen only because of its low mean cloud coverage. Calar Alto, Villafranca and Aix-en-Provence meet especially high availabilities of $63 \%, 66 \%$ and $68 \%$. Another important fact to consider is the variability of these values. The standard deviation of the availability between the single years $(1990,1995,2000,2004,2005)$ is also listed in Table 2. A low value is preferable to keep the availability constant between the different years. So an additional criterion of location selection is a low standard deviation of the availability. 

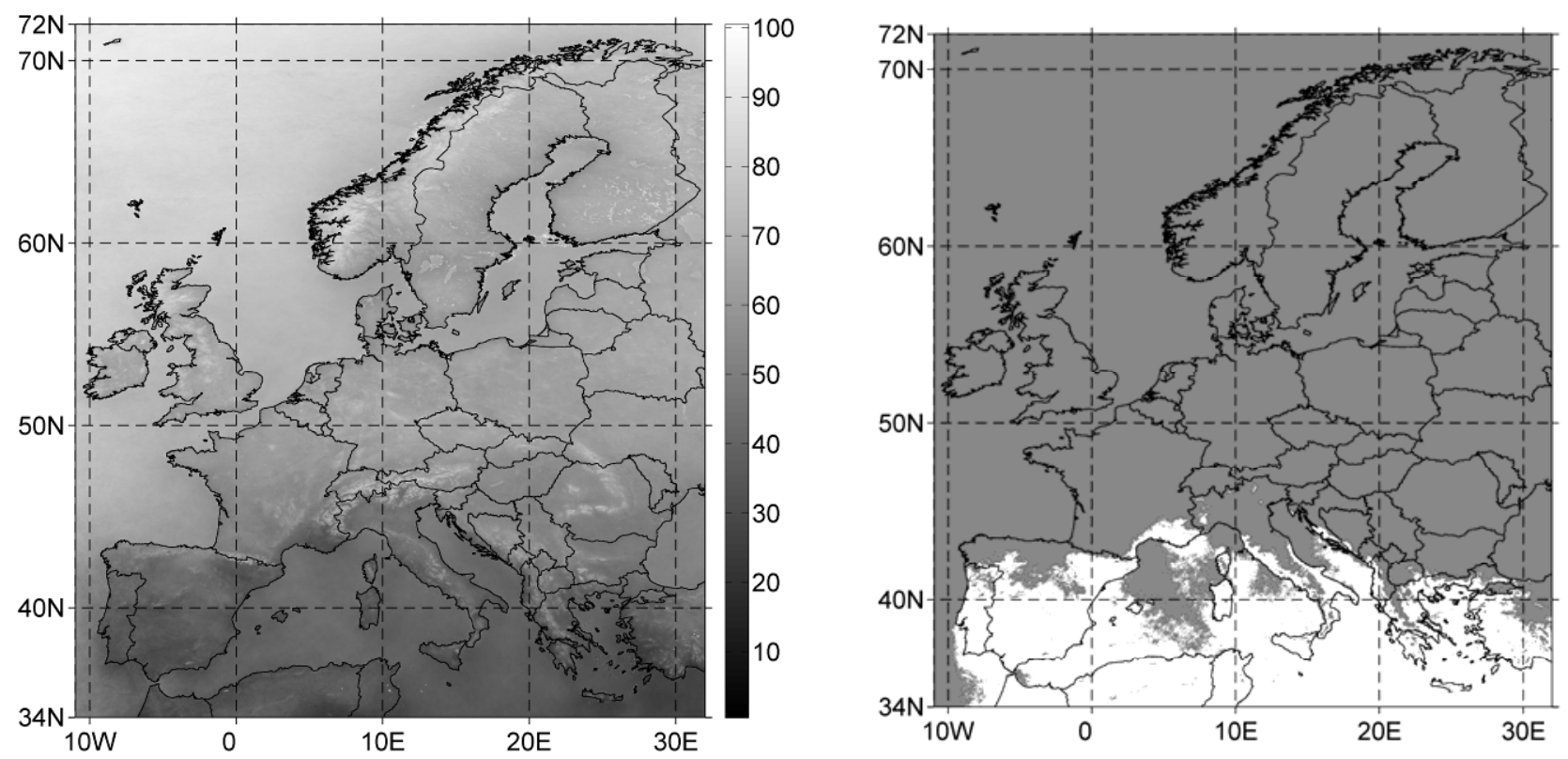

Fig. 7. Mean cloud coverage over Europe derived from ECC data of the years 1990/1995/2000/2004/2005 (left). The typical north-south decrease is clearly visible. Also the influence of mountains can be seen, e.g. the alps and the Carpathians. The right graph shows a binary illustration with a threshold of $60 \%$. The white area depicts an availability of $60 \%$ and more. The gray area corresponds to the availability below $40 \%$.

Table 2. Availability of possible ground station locations.

\begin{tabular}{|l|l|l|l|l|}
\hline Location & Geographic position & Note & Availability [\%] & Standard deviation [\%] \\
\hline Oberpfaffenhofen & $48.080^{\circ} \mathrm{N} 11.280^{\circ} \mathrm{E}$ & DLR & 40 & 6 \\
\hline Neustrelitz & $53.330^{\circ} \mathrm{N} 13.070^{\circ} \mathrm{E}$ & DLR & 37 & 4 \\
\hline Kiruna & $67.857^{\circ} \mathrm{N} 20.964^{\circ} \mathrm{E}$ & ESA & 33 & 9 \\
\hline Calar Alto & $37.220^{\circ} \mathrm{N} 2.546^{\circ} \mathrm{W}$ & Observatory & 63 & 11 \\
\hline Villafranca & $40.440^{\circ} \mathrm{N} 3.950^{\circ} \mathrm{W}$ & ESA & 66 & 9 \\
\hline Aix-en-Provence & $43.531^{\circ} \mathrm{N} 5.444^{\circ} \mathrm{E}$ & - & 68 & 5 \\
\hline
\end{tabular}

Fig. 7 and Table 2 refer to the respective yearly mean. Thus seasonal effects between summer and winter months completely vanish. In analogy to the calculation of $P(W)_{\text {Year }}$ the mean coverage of the single quarters of the year $P(W)_{\text {Quarter }}$ are determined. The quarters are separated as follows:

Quarter 1

January, February, March

\section{Quarter 2}

April, May, June
Quarter 3

July, August, September

\section{Quarter 4}

October, November, December

The coverage maps for the quarters are shown in Fig. 8. As it is expected the cloud coverage in the beginning and the end of the year is higher than in the middle. Aix-en-Provence for instance seems to be a good choice with its availability of $68 \%$. But going through the quarters of the year, varying availabilities of $64 \%, 71 \%, 72 \%$ and $66 \%$ are observed. For the stations in Table 2 the availabilities for the single quarters are listed in Table 3. All stations show a similar variability of $P(V)_{\text {Quarter. }}$. The best time of the year seems to be Quarter 3, followed by Quarter 2, Quarter 1 and 4.

Table 3. Availability [\%] of possible ground station locations for the four quarters of the year.

\begin{tabular}{|l|l|l|l|l|}
\hline Location & Quartal 1 & Quartal 2 & Quartal 3 & Quartal 4 \\
\hline Oberpfaffenhofen & 30 & 47 & 54 & 29 \\
\hline Neustrelitz & 23 & 46 & 46 & 32 \\
\hline Kiruna & 24 & 37 & 45 & 26 \\
\hline Calar Alto & 63 & 62 & 78 & 52 \\
\hline Villafranca & 61 & 69 & 85 & 50 \\
\hline Aix-en-Provence & 64 & 71 & 72 & 66 \\
\hline
\end{tabular}



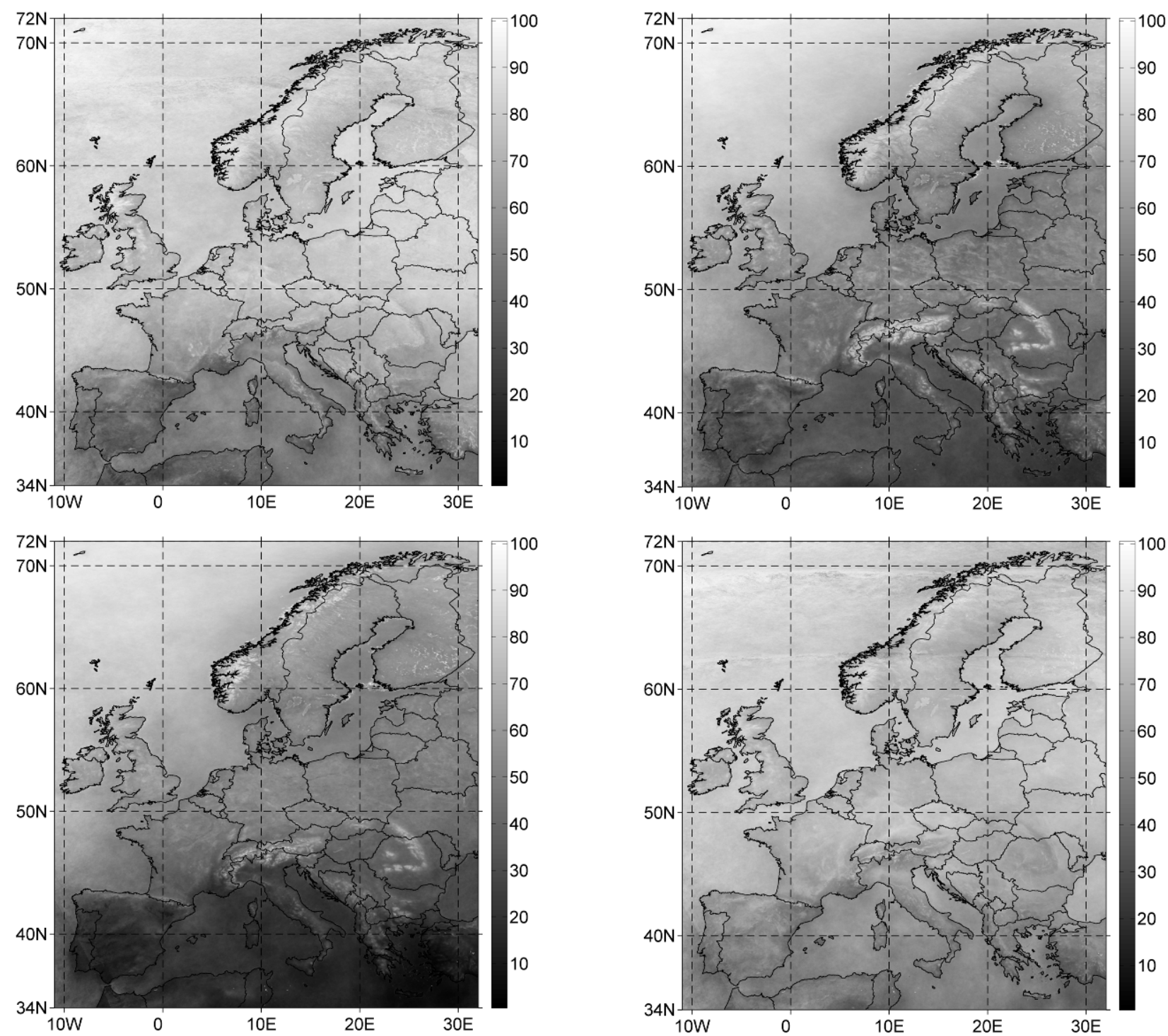

Fig. 8. Mean cloud coverage over Europe derived from ECC data for the four Quarters of the year. From the upper left to down right: Quarter 1, Quarter 2, Quarter 3 and Quarter 4. The seasonal variation is clearly visible. Quarter 3 seems to be the best time of the year for optical downlinks.

\subsection{Availability of ground station diversity systems}

To raise the availability one can set up a ground station diversity system. The more ground stations are used the higher becomes the system availability. Two important facts have to be considered. First is of cause to use stations with low cloud coverage, second is to choose locations whose weather cells are as much uncorrelated as possible. In most cases the correlation decreases with higher distances. Since the single satellite images don't cover the whole ECC map, not every image can be used for joint availability calculation. On a day where no image is found that contains all regarded station locations, a new image is built with multiple original images. Because the different years contain a different number of images first yearly means are calculated and afterwards a total mean to avoid overweighting a single year. Two methods for the calculation of joint availabilities are possible. Either the stations are located very far away from each other, so that the correlation can be neglected or the correlation has to be taken into account. Both methods are 
compared in the following with two exemplary scenarios with four ground stations respectively. The first one is located in Germany, where the stations have a distance between $300 \mathrm{~km}$ and $1000 \mathrm{~km}$ to each other. The second one is distributed over Europe whereas all distances are bigger than $900 \mathrm{~km}$. Here the locations are chosen with respect to low cloud coverage and infrastructural accessibility. The locations are: Calar Alto (1), Aix-en-Provence (2) and places nearby Catania (3) and Heraklion (4). The geographical location of the two scenarios is depicted in Fig. 9.
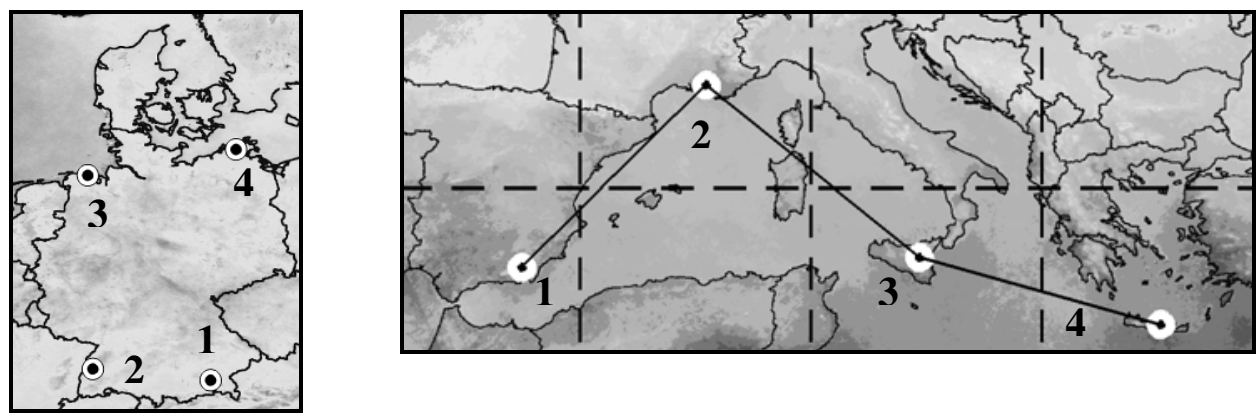

Fig. 9. Locations of the stations in the two exemplary scenarios. On the left is the scenario in Germany. Here the distances of the stations lie between $300 \mathrm{~km}$ and $900 \mathrm{~km}$. On right the one in Europe, where the stations have a least distance of $1000 \mathrm{~km}$ to each other.

The mathematical description of the two methods (neglecting and considering correlation) is quite simple:

\section{Method I:}

This method assumes statistical independency of the single stations. The joint availability $P(V)_{\text {uncorr }}$ is then calculated with

$$
P(V)_{\text {uncorr }}=1-\prod_{k=1}^{M}\left(\frac{1}{N} \sum_{i=1}^{N} P\left(W_{k}\right)_{i}\right) .
$$

Whereas $M$ is the number of used stations and $P\left(W_{\mathrm{k}}\right)$ is the mean coverage in a single year with number of years $N$. So at first the single mean cloud coverage for every location is determined. Then the joint mean cloud coverage is gained by multiplication. Finally the availability results after subtraction from one.

\section{Method II:}

To factor in the correlation first the joint frequency of cloud coverage for the individual years is calculated. Let $M$ again be the number of stations and $J$ the number of images in a year. $P\left(W_{\mathrm{A}, k}\right)$ is the cloud coverage of one day of the $k$-th station. Then the joint frequency of cloud coverage for one year is calculated with

$$
P(W)_{\text {joint }}=\frac{1}{K} \sum_{j=1}^{J}\left(\prod_{k=1}^{M} P\left(W_{A, k}\right)\right)_{j} .
$$

The inner product calculates the joint cloud coverage on one day. Then the mean of all days in one year is determined.

With $N$ as the number of years the availability of the system $P(V)_{\text {corr }}$ is calculated with

$$
P(V)_{\text {corr }}=1-\frac{1}{N} \cdot \sum_{i=1}^{N} P(W)_{\text {joint }, i} .
$$

The raise of availability for both scenarios and methods is depicted in Fig. 10. The more stations are used the higher the availability becomes. Looking at the scenario in Europe the difference in availability between the two methods is very low. The reason is the big distance between the locations and thus a negligible correlation. The order of adding the stations is 4-2-3-1. Only by using the station near Heraklion the availability is almost $75 \%$. With only two stations over $90 \%$ is achieved and with the further two stations availability approaches $99 \%$. 
The system in Germany behaves differently. The adding of the stations is 4-1-3-2. Because the stations are closer, the correlation is not negligible any more. With four stations the difference of availability between method I and II is around $8 \%$. However all four stations together reach an availability of $82 \%$. Adding more stations to the German system will increase the correlation and thus the raise of availability diminishes. Another advantage of multiple ground stations is the lower deviation of the mean value of availability. The right graph in Fig. 10 shows a general decrease on the standard deviation of the availability of the single years. So the availability becomes more constant. The slight increase of the standard deviation with three stations in Germany is caused by the influence of the correlation.
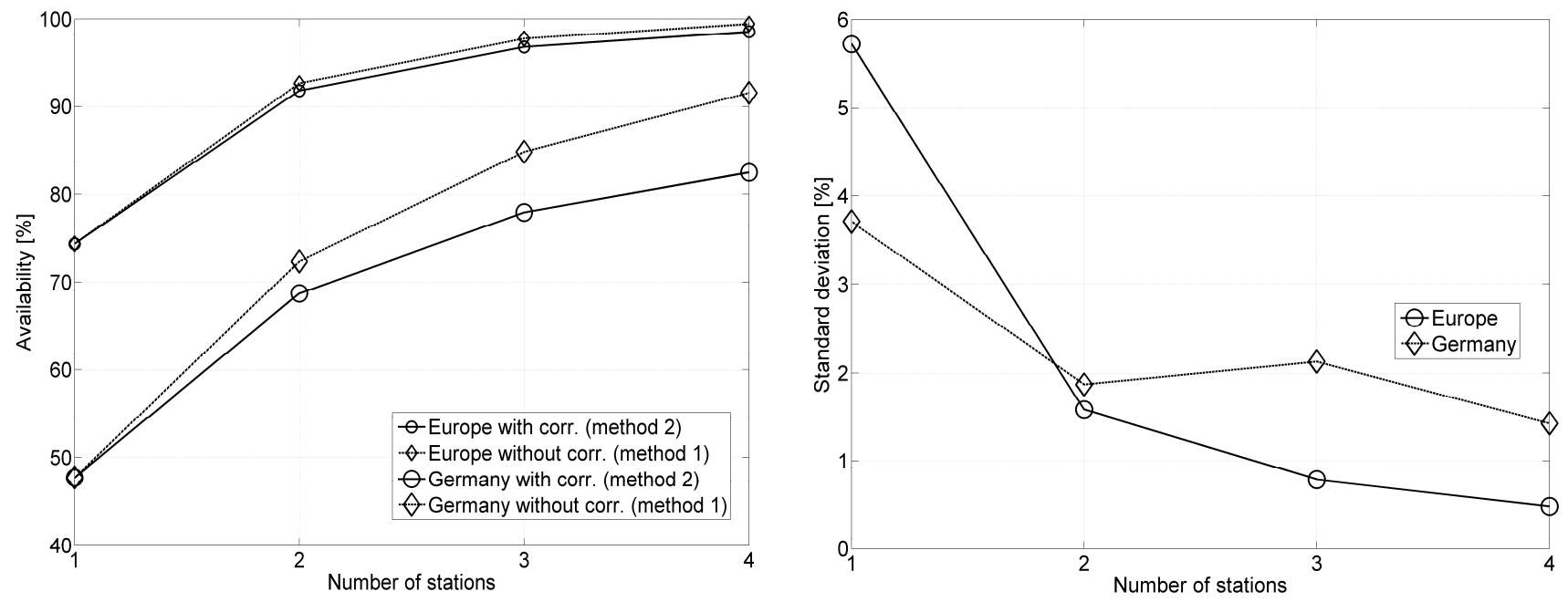

Fig. 10. Increase of availablity of a ground station diversity systems in Europe and Germay (left). The dashed lines show the availability when correlation between weather cells is neglected, the solid lines when correlation is taken care of. The right graph illustrates an decrease in variability of the availability with an increasing number of stations.

\section{CONCLUSION}

The goal of this paper has been to characterize the problem of optical links through the atmosphere when cloud attenuation is involved and to suggest methods to improve the availability of these links. So the wavelength selection criterion of lower cloud attenuation was of major concern. The transmission of clouds has been studied which resulted in a proposal to use wavelengths between $10 \mu \mathrm{m}$ and $12 \mu \mathrm{m}$ to increase the availability of a FSOC system. The atmospheric transmission of this spectral interval has been investigated to attest a possible use. The increase of availability is expected to be very low. Thus a statistical analysis of satellite images of cloud coverage over Europe was done and favorable locations were identified exemplary. An application of ground station diversity was presented to illustrate the possible increase of system availability.

In the NIR all wavelengths approximately suffer from the same attenuation which is very high for most types of water clouds. The attention is directed to wavelengths in the MIR between $10 \mu \mathrm{m}$ and $12 \mu \mathrm{m}$. It was shown that this wavelengths experience much less attenuation than any other wavelength in an atmospheric transmission window. But there is no most suitable wavelength inside this interval because the local attenuation minimum is different for every cloud and thus a tunable laser source should be used. Possible laser sources are $\mathrm{CO}_{2}$-lasers and quantum cascade lasers, which practical use in FSOC systems is currently investigated, but unfortunately there is no off-the-shelf technology satisfying the demands of FSOC available so far. However the attenuation of most cloud types is still too high and thus by applying MIR wavelengths in FSOC only a slight increase in availability is expected. Additionally these long wavelengths should only be applied on short links like HAP-ground to reduce free space loss. These kinds of links are considered for HAPs as relay stations for an increase of down link capacity of earth exploration missions [13].

The molecular absorption of the atmospheric window VIII was calculated in high resolution and shown, that it is widely usable with respect to the strong interrupting absorption lines. For instance the $\mathrm{CO}_{2}$-laser with its $10.6 \mu \mathrm{m}$ is appropriate. 
The statistical evaluation of ECC data is feasible to achieve an estimation of mean availability for either single locations or ground station diversity. With respect to the cloud coverage an OGS is recommended to be placed in southern Europe. The availability is not constant for every year. This is described with the standard deviation of the availability of the single years and can be quite high, e.g. $9 \%$ and $11 \%$ in the case of Kiruna, Calar Alto and Villafranca. So an estimation of availability can only be made for a long period of time since mean coverage of single years can be quite different. This difference of availability between the single years also decreases with the station diversity which can be seen with the lower standard deviation. Another important effect to consider is the seasonal dependency of the cloud coverage. Quarter 3 seems to have the highest availability for most of the regions. Availability can be estimated for stationary links (GEO-GND, HAP-GND). It behaves different with LEO-GND links because in this case the overfly time of the LEO must be taken into account. An example of a ground station diversity system with four stations distributed over Europe is presented and an availability of almost $99 \%$ is possible.

To confirm the results of the availability further statistics with data from more years must be calculated in future. Additionally other data bases with comparable spatial resolution can be used for confirmation, e.g. data from the Moderate Resolution Imaging Spectroradiometer (MODIS) or Meteosat Second Generation (MSG). To get information about wavelength dependent availability the optical depth derived from satellite images can be used for a more sophisticated statistical analysis. But at the time of investigation no data base containing satellite images of wavelength dependent optical depth was available.

\section{ACKNOWLEDGEMENTS}

Thanks go to the Deutsches Fernerkundungsdatenzentrum (DFD), institute of the German Aerospace Center, which supplied all ECC data and G. Gesell (DFD) who gave personal help.

\section{REFERENCES}

1. W. Pratt, Laser Communication Systems. New York: John Wiley \& Sons, Inc., 1969.

2. A. Kylling and B. Mayer, "Technical note: the libRadtran software package for radiative transfer calculations description and examples of use," in Atmospheric Chemistry and Physics Discussions, 2005, pp. 1855-1877.

3. D. Deirmendjian, Electromagnetic Scattering on Spherical Polydispersions. New York: American Elsevier, 1969.

4. K. N. Liou, Y. Takano, S. C. Ou, M. W. Johnson, "Laser transmission through thin cirrus clouds, "Applied Optics, vol. 37, no. 27, pp. 4886-4894, September 2000.

5. E. P. Shettle, "Models of Aerosols, Clouds and Precipitation for Atmospheric Propagation Studies," in Conference Proceedings of AGARD No. 454, Atmospheric Propagation in the UV, Visible, IR and MM-wave region and related system aspects, 1989, pp. 15-1-15-13.

6. K. N. Liou, Radiation and Cloud Processes in the Atmosphere. Oxford: Oxford University Press, 1992.

7. World Meteorological Organization, International Cloud Atlas Vol. 1, Geneva, 1975.

8. J. H. Churnside, K. Shaik, "Atmospheric Propagation issues relevant to optical communications," NOAA Technical Memorandum ERL WPL-159, Boulder, Colorado, 1989.

9. H. C. Van De Hulst, Light Scattering by Small Particles. New York: John Wiley \& Sons Inc, 1957.

10. D. P. Edwards, "GENLN2: A General Line-by-Line Atmospheric Transmittance and Radiance Model, Version 3.0 Description and Users Guide," National Center for Atmospheric Research, Colorado, 1992.

11. The World Data Center for Remote Sensing of the Atmosphere, German Aerospace Center (DLR), "Cloud physical products of latest AVHRR-scenes received in Oberpfaffenhofen," July 2007, http://wdc.dlr.de/apollo/index.html.

12. R. Meerkötter, C. König, P. Bissoli, G. Gesell, H. Mannstein, "A 14-year European Cloud Climatology from NOAA/AVHRR data in comparison to surface observations," Geophysical Research Letters, vol. 31, 2004.

13. M. Knapek, J. Horwath, D. Giggenbach, B. Epple, H. Bischl, N. Courville, F. Moll, "Optical High-Capacity Satellite Downlinks via High-Altitude Platform Relays," to be published at the SPIE Free-Space Laser Communications VII, San Diego, Aug. 2007. 